\title{
A SEMI-THEORETICAL MODEL FOR TRANSFORMATION PLASTICITY OCCURRED DURING BAINITIC TRANSFORMATION
}

\author{
Mahmoud YaAkoubi, Fakhreddine Dammak \\ Laboratory of Mechanics, Modeling and Production (LA2MP), ENIS, Sfax, Tunisia \\ e-mail:mahmoud5yakoubi@gmail.com; fakhreddine.dammak@enis.rn.tn
}

\begin{abstract}
This study addresses the task of predicting the transformation plasticity induced during phase transformation of the 16MND5 carbon steel from austenite to bainite under low externally applied stress using a semi-theoretical model based on the Greenwood-Johnson mechanism. Both models proposed by Leblond et al. (1989) and Taleb and Sidoroff (2003) sufficiently describe the evolution of the TRansformation Induced Plasticity (TRIP) during continuous cooling of the austenitic phase. Nevertheless, TRIP values predicted by these models underestimate measured data through the first half of the transformation and overestimate them through the second half. So, we propose in this paper a method to improve Taleb's model in order to remove discrepancies between theoretical and experimental results throughout the whole transformation and obtain a better description of experimental data.
\end{abstract}

Keywords: bainitic transformation, transformation induced plasticity (TRIP), traction loading

\section{Introduction}

In some thermo-mechanical manufacturing processes, especially heat treatment and welding, phase transformations can occur and can affect significantly the mechanical behavior and structural properties of quenched or welded steel parts. Indeed, when metallurgical transformations occur under small external stress lower than the yield stress of the weaker phase (austenite), a supplementary plastic strain is observed (Leblond et al., 1986, 1989; Fischer et al., 1998; Taleb et al., 2001; Taleb and Sidoroff, 2003; Meftah et al., 2007; Hoang et al., 2008; Moumni et al., 2011). This plastic strain increment is called TRansformation Induced Plasticity (TRIP) and has a significant effect on the distribution of the residual stresses, distortions and mechanical properties (Leblond et al., 1986, 1989; Taleb et al., 2004; Dan et al., 2008; Tahimi et al., 2012; Deng and Murakawa, 2013; Song et al., 2014). In the literature, there are two mechanisms proposed to explain the origin of the TRIP: Magee's mechanism which is proposed for displacive transformations and Greenwood-Johnson's mechanism which is well suitable for the diffusional transformations (Meftah et al., 2007; Hoang et al., 2008). According to Magee, TRIP is due to privileged orientation of martensitic plates during transformation in presence of external stress. While Greenwood and Johnson supposed that accommodation between differences in compactness and dilation coefficients of the parent and the product phase leads to apparition of local dislocations in the vicinity of the interface between phases. When deviatoric stress is applied, dislocations are oriented in the direction of the applied stress which induces transformation plasticity at the macroscopic scale. However, it was revealed through experimental analysis that Magee's mechanism was not dominant for low applied stresses; hence, it was admitted that Magee's mechanism might not be considered for carbon steels; however it is normally deemed for shape memory alloys (Moumni et al., 2011). In addition, it was illustrated that only Greenwood-Johnson's mechanism was considered when modeling TRIP for both diffusional and shear transformations (Taleb et al., 2001). 
It is well known that much works have been done in the last thirty years for better modeling of the evolution of TRIP during phase transformations of steel alloys under different types of loadings. So this additional strain increment originating from phase transformation is accounted in the development of constitutive behavior of a multiphasic material in order to perfectly simulate the material response in continuum mechanical computation. The approaches describing the evolution of the TRIP during phase transformations can be classified into phenomenological models (Mohr and Jacquemin, 2008), micromechanics-based models (Leblond et al., 1989; Taleb and Sidoroff, 2003; Sun et al., 2009) and discrete dislocation-transformation model (Shi et al., 2010).

In this paper, we focus only on the micromechanics-based model proposed by Leblond et al. (1989) and improved later by Taleb and Sidoroff (2003). This theoretical model was established by considering Greenwood-Johnson's mechanism where an elementary volume of austenite having spherical shape in which a spherical core of $\alpha$-phase was growing. The homogeneity of strain and stress fields in the transforming elementary volume was assumed. So, micromechanical analysis permits establishing the theoretical model describing TRIP during phase transformation.

The purpose of this work is to present a summarization of the hypothesis and theoretical development carried out by the authors to obtain their TRIP models, appraise their simulation results, and improve some assumptions resulting then in a better model which predicts effectually the evolution of the TRIP during phase transformation. Simulations obtained by the new model will be compared with experimental results provided in the literature in order to investigate the efficiency of our modeling.

\section{Basic framework}

We are interested in the theoretical model developed by Leblond et al. (1989) which is one of the most widely used for practical applications and which is implemented in the finite element codes such as SYSWELD and ASTER. This model was improved later by Taleb and Sidoroff (2003).

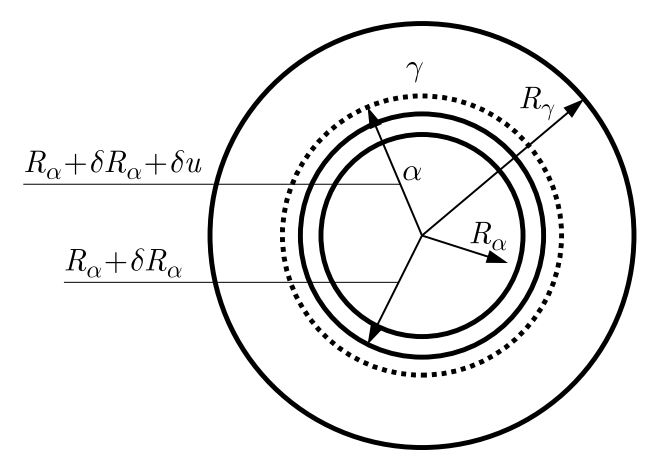

Fig. 1. Geometry considered by Leblond to illustrate phase transformation of austenitic nuclei

Leblond's model is obtained from a micromechanical analysis of stress and strain fields which evolve in an austenitic spherical nuclei occurring during continuous cooling. The growth of a spherical product phase core is carried out in the center of austenitic spherical nuclei as shown in Fig. 1. $R_{\gamma}$ and $R_{\alpha}$ are radii of parent and product phase, respectively. One should note that $R_{\alpha}$ is nil before the beginning of transformation and it grows progressively during transformation until it reaches $R_{\gamma} . \delta R_{\alpha}$ is the radius increasing of the spherical phase $\alpha$ during a time increment $\delta t$. Because of the positive volume change induced by the transformation, points located originally at $R_{\alpha}+\delta R_{\alpha}$ come to a new location $R_{\alpha}+\delta R_{\alpha}+\delta u$. As revealed by Leblond, the macroscopic plastic strain rate generated during phase transformation under external loading depends only 
on the shape variation of each phase. Indeed, the author assumed through its hypothesis 1 that the effect of local anisotropy due to a small difference between elastic parameters of each phase is negligible with respect to the stresses and deformations due to volume differences between phases $\alpha$ and $\gamma$. So, the general expression for the plastic strain rate is given by the following equation

$$
\dot{E}^{p}=(1-z)\left\langle\dot{\varepsilon}_{\gamma}^{p}\right\rangle_{V_{\gamma}}+z\left\langle\dot{\varepsilon}_{\alpha}^{p}\right\rangle_{V_{\alpha}}+\dot{z}\left\langle\Delta \varepsilon_{\gamma \rightarrow \alpha}^{p}\right\rangle_{F}
$$

where $z$ is the volume fraction of the product phase, $\dot{\varepsilon}_{\gamma}^{p}$ and $\dot{\varepsilon}_{\alpha}^{p}$ are the microscopic plastic strain rate tensors in phases $\gamma$ and $\alpha$, respectively, $\Delta \varepsilon_{\gamma \rightarrow \alpha}^{p}$ is the deviatoric component of the transformation strain tensor and $\left\langle\Delta \varepsilon_{\gamma \rightarrow \alpha}^{p}\right\rangle_{F}$ expresses the average value of $\Delta \varepsilon_{\gamma \rightarrow \alpha}^{p}$ along the transformation front $F$.

The author assumed that the average of deviatoric transformation strain tensor on the front $F$ is negligible since there is no favorite orientation. Subsequently, the last term in equation (2.1) is omitted. The second hypothesis proposed by the author is that for small or moderately high applied stresses, the austenitic phase is entirely plastic, but the $\alpha$-phase remains elastic or its plastic strain rate remains always much smaller than that of the $\gamma$-phase. Afterward, the second term in the right-hand side of equation (2.1) disappears, and this later is reduced to

$$
\dot{E}^{p}=(1-z)\left\langle\dot{\varepsilon}_{\gamma}^{p}\right\rangle_{V_{\gamma}}
$$

Given that the plastic strain in the parent phase is the sum of the classical plastic term due to variation of the loading conditions and transformation induced plastic term corresponding to the evolution of new phase fraction $z$, then

$$
\dot{E}^{p}=\dot{E}^{c p}+\dot{E}^{t p}
$$

$\dot{E}^{c p}$ is the classical plastic term and the transformation induced plastic term is written as follows

$$
\dot{E}^{t p}=(1-z)\left\langle\frac{\delta \varepsilon_{\gamma}^{p}}{\delta z}\right\rangle_{V_{\gamma}} \dot{z}
$$

The third hypothesis used by the author is that material obeys the Von Mises criterion and possesses an ideal-plastic flow. By assuming a uniform austenitic yield stress $\sigma_{\gamma}^{y}$, equation (2.4) can be transformed into

$$
\dot{E}^{t p}=\frac{3(1-z)}{2 \sigma_{\gamma}^{y}}\left\langle\frac{\delta \varepsilon_{\gamma}^{e q}}{\delta z} s_{\gamma}\right\rangle_{V_{\gamma}} \dot{z}
$$

where $\varepsilon_{\gamma}^{e q}$ is the von Mises equivalent microscopic plastic strain in the parent phase (phase $\gamma$ ), $\sigma_{\gamma}^{y}$ and $\mathbf{s}_{\gamma}$ are respectively the yield stress and the deviatoric tensor of the microscopic stress in this phase.

At this stage, Leblond assumed through hypothesis 4 and 5 that correlation between $\delta \varepsilon_{\gamma}^{e q} / \delta z$ and $s_{\gamma}$ can be neglected and the average $S_{\gamma}$ of $s_{\gamma}$ within the volume $V_{\gamma}$ is equal to the overall average $S$ of $s$ in the whole nuclei. Then

$$
\dot{E}^{t p}=\frac{3(1-z)}{2 \sigma_{\gamma}^{y}}\left\langle\frac{\delta \varepsilon_{\gamma}^{e q}}{\delta z}\right\rangle_{V_{\gamma}} S \dot{z}
$$

Using a spherical coordinate system and considering a purely radial displacement, the solution of the mechanical problem is performed using the dynamic equilibrium equation in the continuous mediums. Finally, it is found that

$$
\frac{\delta \varepsilon_{\gamma}^{e q}}{\delta z}=\frac{2 \Delta \varepsilon_{\alpha \gamma} R_{\gamma}^{3}}{r^{3}}
$$


So

$$
\dot{E}^{t p}=-\frac{3 \Delta \varepsilon_{\alpha \gamma}}{\sigma_{\gamma}^{y}} \ln (z) \dot{z} S
$$

where $\Delta \varepsilon_{\alpha \gamma}$ is the volume change that corresponds to phase transformation.

Because equation (2.8) includes a singularity at the beginning of the transformation $(z=0)$, the author proposed to cut off the TRIP below $z=0.03$ leading then to the following model

$$
\dot{E}^{t p}= \begin{cases}0 & \text { if } \quad z \leqslant 0.03 \\ -\frac{3}{2} k \ln (z) \dot{z} S & \text { if } \quad z>0.03\end{cases}
$$

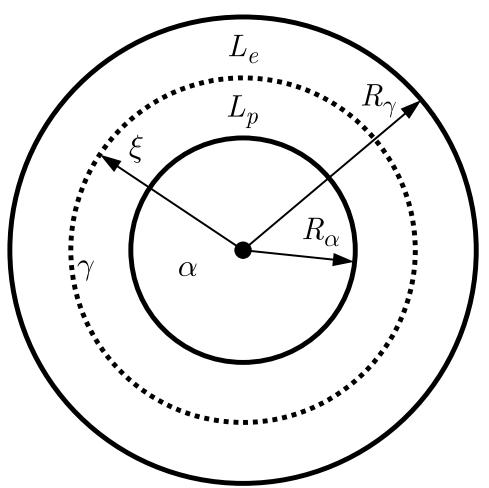

Fig. 2. Geometry considered by Taleb and Sidoroff (2003): $\gamma$-phase is composed by an outer elastic layer $L_{e}$ around an inner plastic layer $L_{p}$

Taleb and Sidoroff (2003) developed their model by following the micromechanical scheme assumed by Leblond to formulate its transformation plasticity kinetic model. They extended the Leblond model by keeping all hypotheses except hypothesis 2. Afterwards, the behavior of the austenitic phase has been considered elastoplastic with ideal plasticity. Indeed, according to Taleb and Sidoroff (2003), the product phase remains elastic while the parent phase consists of an outer elastic layer $L_{e}$ around an inner plastic layer $L_{p}$ with an elastic-plastic boundary at $r=\xi$ where $R_{\alpha} \leqslant \xi \leqslant R_{\gamma}$ as shown in Fig. 2. The boundary between these layers increases progressively during transformation until $\xi$ becomes equal to $R_{\gamma}$. At this instant, the remainder of the parent phase turns into completely plastic. By executing the solution of the mechanical problem using the dynamic equilibrium equation in the continuous medium, Taleb and Sidoroff (2003) found that

$$
\xi=\sqrt[3]{\frac{2 \Delta \varepsilon_{\gamma \alpha}}{\sigma_{\gamma}^{y}} \frac{9 K \mu}{4 \mu+3 K}} R_{\alpha}
$$

where $K$ and $\mu$ are respectively the bulk and shear elastic moduli.

Finally, Taleb's model assuming elastoplastic parent phase and extending Leblond's one to low values of $z$; is the following

$$
\dot{E}^{t p}= \begin{cases}-\frac{2 \Delta \varepsilon_{\gamma \alpha}}{\sigma_{1}^{y}} \ln \left(z_{\ell}\right) \dot{z} \frac{3}{2} S & \text { if } \quad z \leqslant z_{\ell} \\ -\frac{2 \Delta \varepsilon_{\gamma \alpha}}{\sigma_{1}^{y}} \ln (z) \dot{z} \frac{3}{2} S & \text { if } \quad z>z_{\ell}\end{cases}
$$

with

$$
z_{\ell}=\frac{\sigma_{\gamma}^{y}}{2 \Delta \varepsilon_{\alpha \gamma}} \frac{4 \mu+3 K}{9 K \mu}
$$


Experimental tests on bainitic transformation of the 16MND5 steel under small applied stresses were performed and their results were given by Taleb and Sidoroff (2003). These tests allow comparison between simulation and experiment results. Indeed, theoretical and experimental curves are presented in Fig. 3 providing the evolution of the TRIP against the product phase fraction. So, a coincidence between Taleb's and Leblond's models is observed when the threshold of Leblond's model is equal to 0.03 . Theoretical prediction given by Taleb's model that agrees with Leblond's forecast (Leblond 0.03) illustrates slow transformation plasticity kinetic during the first half of the transformation with respect to the experimental result while the latter seems overestimated at the end of the transformation. Therefore, the aim of the following Section is to revise some assumptions made by the authors; that lead to formulate enhanced transformation plasticity kinetics ensuring then a better congruence with the experimental curve through all the transformation.

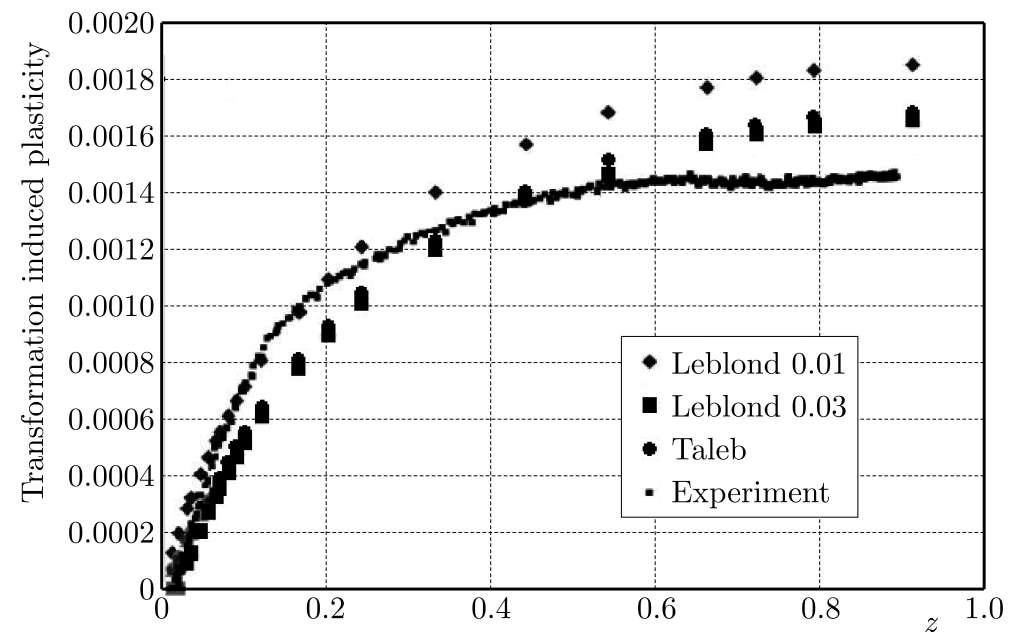

Fig. 3. Transformation plasticity evolution during bainitic transformation in the 16MND5 steel under applied stress $(24 \mathrm{MPa})$ versus volume fraction of the formed bainite

\section{Numerical procedure}

Our new model will be developed basing on the micromechanical analysis presented above after reviewing some assumptions made by the authors. Indeed, some hypothesis will be more discussed and improved leading thus to a more refined model that better agrees with the experimental results.

Hypothesis 4 suggested by the author which assumes that

$$
\left\langle\frac{\delta \varepsilon_{\gamma}^{e q}}{\delta z} s_{\gamma}\right\rangle_{V_{\gamma}}=\left\langle\frac{\delta \varepsilon_{\gamma}^{e q}}{\delta z}\right\rangle_{V_{\gamma}}\left\langle s_{\gamma}\right\rangle_{V_{\gamma}}
$$

is mathematically inaccurate because the integral of the product of two functions is different to the product of their integrals. So, we suppose that the previous equation can be calibrated by introducing a function $m(z)$ as follows

$$
\left\langle\frac{\delta \varepsilon_{\gamma}^{e q}}{\delta z} s_{\gamma}\right\rangle_{V_{\gamma}}=m(z)\left\langle\frac{\delta \varepsilon_{\gamma}^{e q}}{\delta z}\right\rangle_{V_{\gamma}}\left\langle s_{\gamma}\right\rangle_{V_{\gamma}}
$$

In order to have an idea about the evolution of the function $m(z)$, let us consider Fig. $4 \mathrm{a}$ which is available in Leblond et al. (1989) that points out the simulation of $\left\langle\left(\delta \varepsilon_{\gamma}^{e q} / \delta z\right) s_{\gamma}\right\rangle_{V_{\gamma}}$ and $\left\langle\delta \varepsilon_{\gamma}^{e q} / \delta z\right\rangle_{V_{\gamma}}\left\langle s_{\gamma}\right\rangle_{V_{\gamma}}$ versus $z$. Basing on this result, we remark that disagreement between the two 
curves is moderately small; thus we admit that the function $m(z)$ is not varying much in the interval $[0,1]$; so we can substitute equation (3.1) by

$$
\left\langle\frac{\delta \varepsilon_{\gamma}^{e q}}{\delta z} s_{\gamma}\right\rangle_{V_{\gamma}} \approx\langle m(z)\rangle_{[0,1]}\left\langle\frac{\delta \varepsilon_{\gamma}^{e q}}{\delta z}\right\rangle_{V_{\gamma}}\left\langle s_{\gamma}\right\rangle_{V_{\gamma}}
$$

Then hypothesis 4 is replaced by hypothesis 4', thinking that

$$
\left\langle\frac{\delta \varepsilon_{\gamma}^{e q}}{\delta z} s_{\gamma}\right\rangle_{V_{\gamma}}=m\left\langle\frac{\delta \varepsilon_{\gamma}^{e q}}{\delta z}\right\rangle_{V_{\gamma}}\left\langle s_{\gamma}\right\rangle_{V_{\gamma}}
$$

with $m$ is the average of the function $m(z)$ in the interval $[0,1]$.

(a)

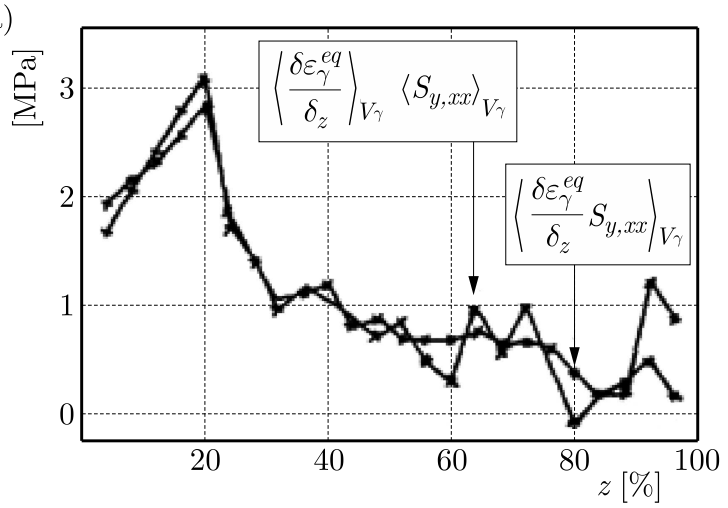

(b)

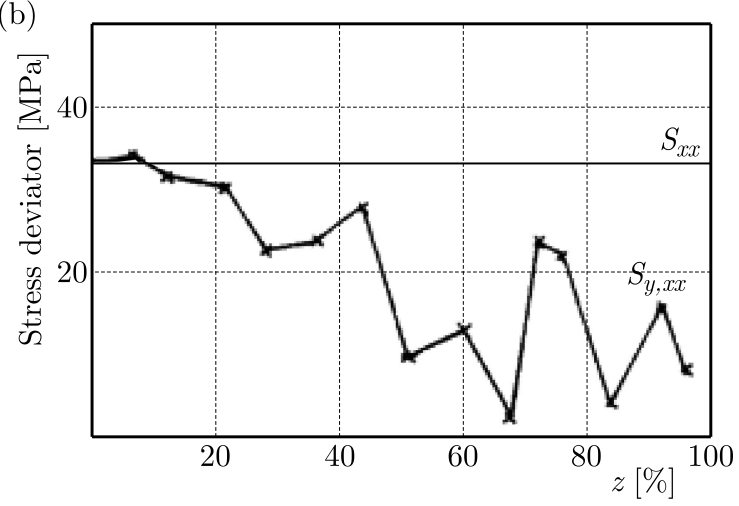

Fig. 4. Verification of: (a) hypothesis 4 and (b) hypothesis 5

Hypothesis 5 assuming that the average stress deviator in the parent phase is almost equal to the overall average stress deviator $\left(S_{\gamma}=S\right.$ with $S_{\gamma}=\left\langle s_{\gamma}\right\rangle_{V_{\gamma}}$ and $\left.S=\langle s\rangle_{V}\right)$ is not verified. Indeed, Fig. 4b which is taken from Leblond et al. (1989) illustrates the simulation of $S_{\gamma}$ and $S$ versus the product phase fraction $z$ in the uniaxial case, and an important discrepancy between them has been shown. More precisely, the curves in this figure prove that $S_{\gamma} / S$ is a decreasing function versus $z$. Afterwards, we propose that the function $S_{\gamma} / S$ has a style of $1-z^{n}$ defining then hypothesis 5'. The new relationship between $S_{\gamma}$ and $S$ is the following

$$
S_{\gamma}=\left(1-z^{n}\right) S
$$

with $n$ is a constant.

Replacing hypotheses 4 and 5 by hypotheses 4' and 5' respectively, one can obtain

$$
\left\langle\frac{\delta \varepsilon_{\gamma}^{e q}}{\delta z} s_{\gamma}\right\rangle_{V_{\gamma}}=m\left(1-z^{n}\right)\left\langle\frac{\delta \varepsilon_{\gamma}^{e q}}{\delta z}\right\rangle_{V_{\gamma}} S=\chi(z)\left\langle\frac{\delta \varepsilon_{\gamma}^{e q}}{\delta z}\right\rangle_{V_{\gamma}} S
$$

with

$$
\chi(z)=m\left(1-z^{n}\right)
$$

The function $\chi$ depends on two parameters $m$ and $n$ and should accomplish more coincidence between quantities $\left\langle\left(\delta \varepsilon_{\gamma}^{e q} / \delta z\right) s_{\gamma}\right\rangle_{V_{\gamma}}$ and $\left\langle\delta \varepsilon_{\gamma}^{e q} / \delta z\right\rangle_{V_{\gamma}} S$. Afterwards, the new model of transformation plasticity evolution is defined as follows

$$
\dot{E}^{t p}(z)=\chi(z) \psi(z) \dot{z}
$$

with

$$
\psi(z)=\left\{\begin{array}{lll}
-\frac{2 \Delta \varepsilon_{\alpha \gamma}}{\sigma_{\gamma}^{y}} \ln \left(z_{\ell}\right) \frac{3}{2} S & \text { if } & z \leqslant z_{\ell} \\
-\frac{2 \Delta \varepsilon_{\alpha \gamma}}{\sigma_{\gamma}^{y}} \ln (z) \frac{3}{2} S & \text { if } & z>z_{\ell}
\end{array}\right.
$$


with

$$
z_{\ell}=\frac{\sigma_{\gamma}^{y}}{2 \Delta \varepsilon_{\alpha \gamma}} \frac{4 \mu+3 K}{9 K \mu}
$$

According to the new model, Eq. (3.7), the transformation plasticity increment that corresponds to a product phase increment produced during a time increment is the following

$$
\Delta E^{t p}\left(z_{j}\right)=\chi\left(z_{j}\right) \psi\left(z_{j}\right)(\Delta z)_{j}=\chi\left(z_{j}\right) \Delta T P_{T}\left(z_{j}\right)
$$

where $z_{j}$ is obtained until $j$-th time increment by accounting from the beginning of bainitic transformation $z_{j}=\sum_{i=1}^{i=j}(\Delta z)_{i},(\Delta z)_{j}$ is the increment of the product phase formed during the $j$-th time increment. $\Delta T P_{T}\left(z_{j}\right)$ represents the transformation plasticity increment generated during the $j$-th time increment according to Taleb's model. Indeed, the function $\chi\left(z_{j}\right)$ is defined as a quotient obtained by dividing $\Delta T P_{E x p}\left(z_{j}\right)$ by $\Delta T P_{T}\left(z_{j}\right)$ for a non nil value of $z_{j}$

$$
\chi\left(z_{j}\right)=\frac{\Delta T P_{\operatorname{Exp}}\left(z_{j}\right)}{\Delta T P_{T}\left(z_{j}\right)} \quad z_{j} \neq 0
$$

where $\Delta T P_{E x p}\left(z_{j}\right)$ designates the experimental value of the plasticity transformation increment developed during the $j$-th time increment. $\Delta T P_{E x p}\left(z_{j}\right)$ and $\Delta T P_{T}\left(z_{j}\right)$ are determined graphically from curves illustrated in Fig. 3. $\chi(1)$ is directly equal to zero from equation (3.6). Subsequently, the curve characterizing the evolution of function $\chi$ against $z$ is illustrated in Fig. 5. One can remark then that the function $\chi$ which depends on $m$ and $n$ parameters is

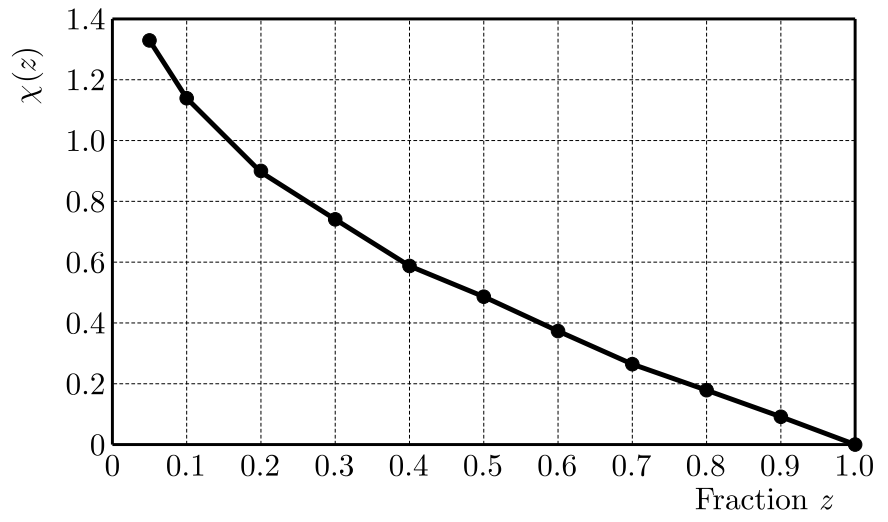

Fig. 5. Evolution of the function $\chi$ versus $z$

decreasing versus $z$. The parameters $m$ and $n$ can be determined by evaluating the derivative of the function $\chi$ for two different values $z_{1}$ and $z_{2}$ as follows

$$
\left.\begin{array}{l}
\chi^{\prime}\left(z_{1}\right)=-m n z_{1}^{n-1} \\
\chi^{\prime}\left(z_{2}\right)=-m n z_{2}^{n-1}
\end{array}\right\} \Rightarrow\left\{n=\frac{\ln \frac{\chi^{\prime}\left(z_{1}\right)}{\chi^{\prime}\left(z_{2}\right)}}{\ln \frac{z_{1}}{z_{2}}}+1 \quad m \approx \frac{1}{2}\left(\frac{\chi\left(z_{1}\right)}{1-z_{1}^{n}}+\frac{\chi\left(z_{2}\right)}{\left.1-z_{2}^{n}\right)}\right.\right.
$$

The estimation of the function $\chi^{\prime}$ at a given value $z$ is accomplished by applying the following formulation

$$
\chi^{\prime}(z)=\frac{\chi(z+\hbar)-\chi(z-\hbar)}{2 \hbar}
$$

with $\hbar$ being a parameter of too small value (it is chosen equal to 0.02 in our case). $\chi(z+\hbar)$ and $\chi(z-\hbar)$ are determined graphically from Fig. 5. It is found that $\chi^{\prime}(0.2)=-1.98$ and $\chi^{\prime}(0.7)=-0.96$. So

$$
n=0.405 \quad m=1.93
$$


Finally, the function $\chi$ is determined

$$
\chi(z)=1.93\left(1-z^{0.405}\right)
$$

According to equations (3.7) and (3.8), the new model for TRIP kinetics, which is formulated to get an improved agreement with the experimental result, is defined by

$$
\dot{E}^{t p}= \begin{cases}-\frac{2 \Delta \varepsilon_{\alpha \gamma}}{\sigma_{\gamma}^{y}} \chi(z) \ln \left(z_{\ell}\right) \dot{z} \frac{3}{2} S & \text { if } \quad z \leqslant z_{\ell} \\ -\frac{2 \Delta \varepsilon_{\alpha \gamma}}{\sigma_{\gamma}^{y}} \chi(z) \ln (z) \dot{z} \frac{3}{2} S & \text { if } \quad z>z_{\ell}\end{cases}
$$

with

$$
\chi(z)=1.93\left(1-z^{0.405}\right) \quad z_{\ell}=\frac{\sigma_{\gamma}^{y}}{2 \Delta \varepsilon_{\alpha \gamma}} \frac{4 \mu+3 K}{9 K \mu}
$$

Now, the new model will be investigated through comparison between numerical simulations and measured TRIP generated during bainitic transformation of 16MND5 steel specimens.

\section{Experimental validation}

In this Section, free dilatometry and TRIP tests carried out by Coret et al. (2002) are deemed. Specimens were 16MND5 steel tubular cylinders having inner and outer diameters equal to $22.4 \mathrm{~mm}$ and $23.4 \mathrm{~mm}$, respectively. The feeble thickness of the specimen enables obtaining low a radial thermal gradient and, subsequently, homogenous stress and strains fields. Specimens were austenitized by induction current at $900^{\circ} \mathrm{C}$ for $30 \mathrm{~s}$ and then cooled by injecting argon inside. The heating and cooling rate were $10^{\circ} \mathrm{C} / \mathrm{s}$ and $-3^{\circ} \mathrm{C} / \mathrm{s}$, respectively. We consider in this paper three experimental results of dilatometric tests provided by Coret et al. (2002). The first was the free dilatometric test while the second and the third were the TRIP dilatometric tests under uniaxial traction loading equal to $30 \mathrm{MPa}$ and $60 \mathrm{MPa}$, respectively. Traction loading was applied during the cooling stage when temperature reached $600^{\circ} \mathrm{C}$ (somewhat before the beginning of bainitic transformation at $560^{\circ} \mathrm{C}$ ) and released at the end of the test. In this study, dilatation curves obtained by these tests were adjusted in such a way that there was no difference between them before reaching temperature $600^{\circ} \mathrm{C}$. In addition, only difference due to elastic strains was considered for temperature between $600^{\circ} \mathrm{C}$ and $560^{\circ} \mathrm{C}$. This procedure takes away experimental uncertainty and allows getting reliable results. Dilatometric curves are plotted together in Fig. 6 for the temperature range $700^{\circ} \mathrm{C}-390^{\circ} \mathrm{C}$ which includes bainitic transformation during cooling. The difference between TRIP curves and free dilatometric curve is due to elastic strain caused by the external loading and essentially to TRIP generated by phase transformation under external stress.

The total strain occurred during a TRIP test is supposed to be the sum of thermo-metallurgical strain, elastic strain due to external loading and plasticity transformation strain generated through phase transformation (Taleb et al., 2001; Coret et al., 2002; Dutta et al., 2013). Then

$$
\varepsilon^{p t}(T)=\varepsilon^{t o t}(T)-\varepsilon^{t h m}(T)-\varepsilon^{e}(T)
$$

with $\varepsilon^{t o t}(T)$ being the total strain issued from TRIP curve. $\varepsilon^{t h m}(T)$ corresponds to strain obtained from the free dilatometric curve and $\varepsilon^{e}(T)$ is the elastic strain. It is given by

$$
\varepsilon^{e}(T)=\frac{\sigma}{E(T)}
$$

where $\sigma$ is the external applied stress and $E(T)$ is the thermal dependent Young modulus. 


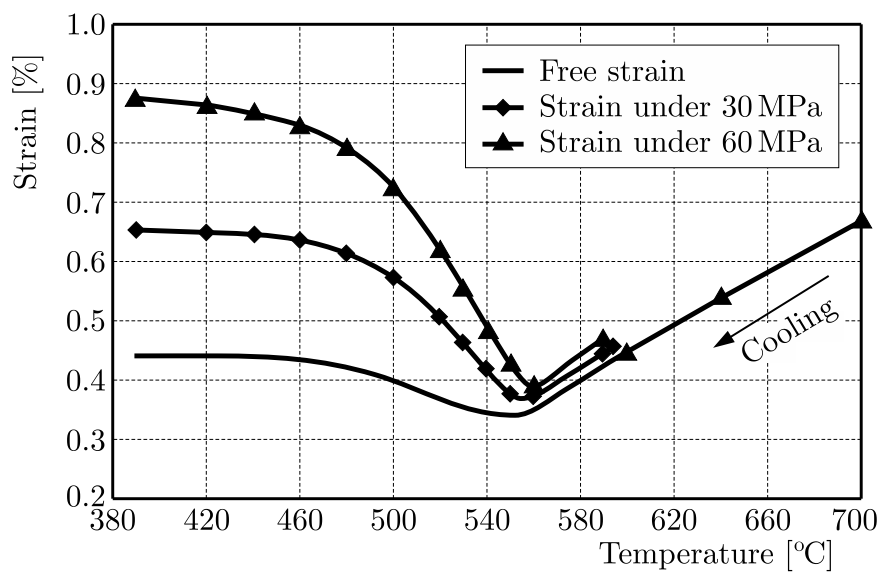

Fig. 6. Dilatometric curves obtained during bainitic transformation under different tension loadings

The evolution of TRIP against the temperature is estimated for two TRIP tests assuming that there is no TRIP evolution before starting of the bainitic transformation. For the 16MND5 steel, when the austenite transforms under cooling rate equal to $-3^{\circ} \mathrm{C} / \mathrm{s}$, the obtained phase proportions are respectively $87 \%$ of bainite and $13 \%$ of martensite (Moumni et al., 2011). Subsequently, we will not take into account of the TRIP occurred below $390^{\circ} \mathrm{C}$ when estimating the evolution of TRIP against temperature because we consider in this study only TRIP occurred through bainitic transformation. Experimental results for the evolution of TRIP according to temperature during cooling are illustrated in Table 1. Now, these experimental results will be used to evaluate numerical simulations performed in the following Section.

Table 1. TRIP obtained from dilatometric tests

\begin{tabular}{|l|c|c|c|c|c|c|c|c|c|}
\hline Temperature $\left[{ }^{\circ} \mathrm{C}\right]$ & 560 & 540 & 520 & 500 & 480 & 460 & 440 & 420 & 390 \\
\hline$\varepsilon^{t p}[\%], \sigma=30 \mathrm{MPa}$ & 0 & 0.052 & 0.118 & 0.152 & 0.169 & 0.182 & 0.188 & 0.192 & 0.194 \\
\hline$\varepsilon^{t p}[\%], \sigma=60 \mathrm{MPa}$ & 0 & 0.099 & 0.212 & 0.290 & 0.335 & 0.359 & 0.378 & 0.390 & 0.402 \\
\hline
\end{tabular}

\section{Simulations and discussions}

The simulation of the quenching process is performed through calculation of temperature evolution in the specimen during treatment. It is coupled with calculation of metallurgical phases distributions followed by the solution of the mechanical problem by the finite element method. The ABAQUS software linked to many subroutines is used to simulate heat treatment phase transformation histories and strain fields as mentioned by Yaakoubi et al. (2013b). The thermal cycle recorded by Coret et al. (2002) is used as the boundary condition to carry out the simulation (Fig. 7). Phase transformation kinetics is modeled by using the JMAK formalism (Barbe et al., 2008; Yaakoubi et al., 2013a). Thermo-physical properties of the material are available in Moumni et al. (2011). The analysis is realized two times for each loading case by using in the first time Taleb's model to predict TRIP evolution and using the new model in the second time. It is found that the maximum value of bainite proportion obtained by simulation is equal to 0.883 , which is very close to value (0.87) obtained by Moumni et al. (2011).

The comparison between numerical and measured TRIP $\left(\varepsilon^{t p}=f(T)\right)$ appears in Fig. 8. It is evident that the transformation plasticity predicted by the new model is considerably better than Taleb's predictions for the loading case of $30 \mathrm{MPa}$. However, for the loading case of $60 \mathrm{MPa}$, new predictions are not adequate because they show overestimations through the first half of the transformation and underestimations through the second half. We observe that as the tension 


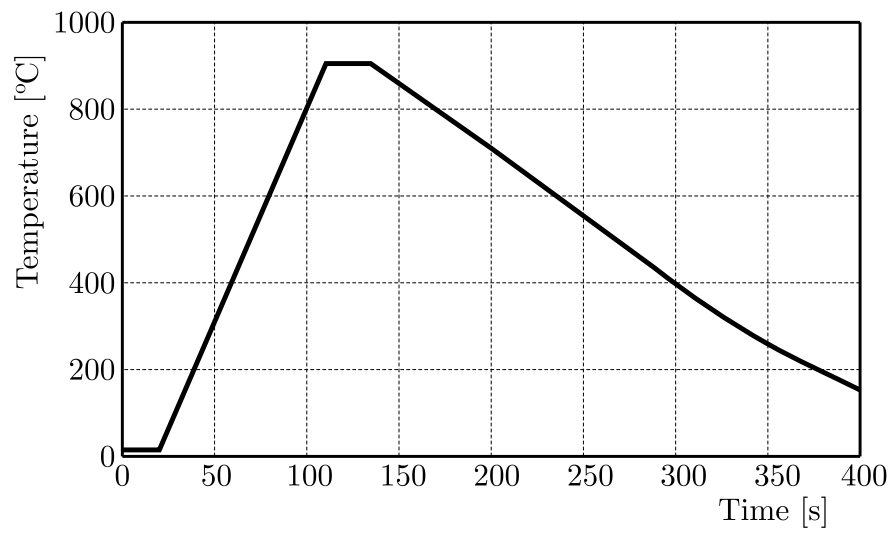

Fig. 7 . Thermal cycle used as the boundary condition to carry out the simulation
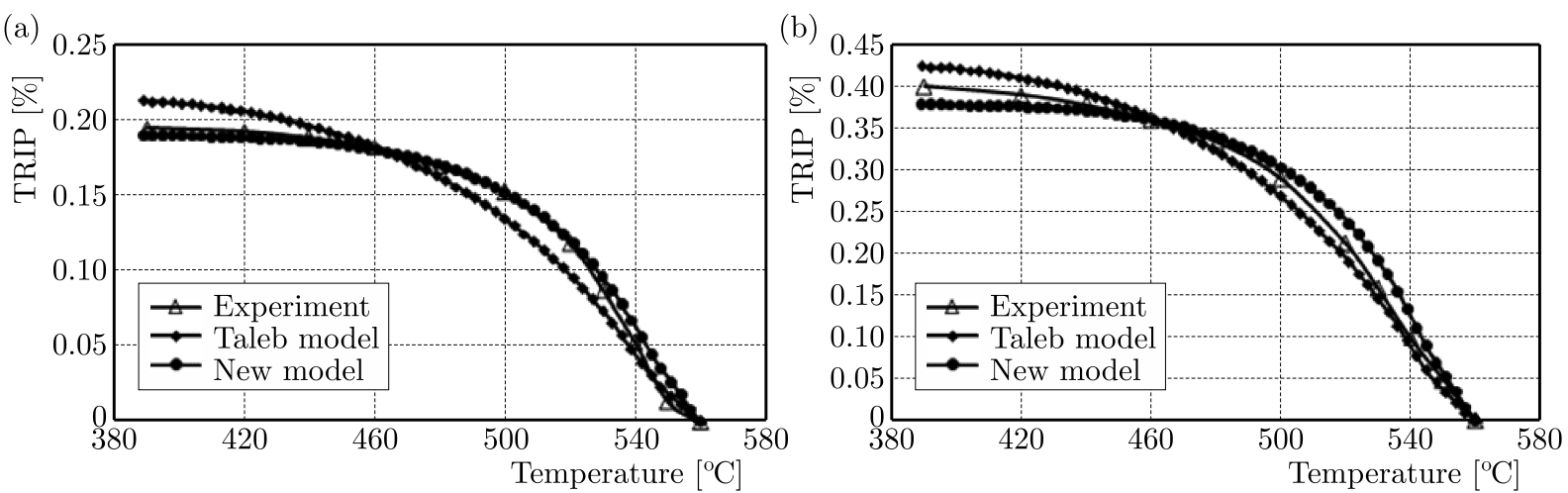

Fig. 8. Transformation plasticity evolution versus temperature during transformation plasticity tests under (a) $30 \mathrm{MPa}$, (b) $60 \mathrm{MPa}$. Comparison between experimental results and predictions of Taleb's and the new model

load becomes larger, the discrepancies of the new model become increasingly significant. This fact is explained by that the function $\chi$ which appears in the new model is identified for the loading case of $24 \mathrm{MPa}$. So, we think that this new model can be refined by making parameters $m$ and $n$ (those define the function $\chi$ ) dependent on the applied stress $\sigma$. Indeed, analysis of the function $\chi$ shows that the increasing of the parameter $m$ increases the predictions of TRIP at the beginning of transformation, and that the increasing of the parameter $n$ increases them at the end of the transformation and vice versa. The fitting of numerical simulations conducted to define parameters $m$ and $n$ versus $\sigma$ is as follows

$$
m=\frac{10}{\sqrt{\sigma+3}} \quad n=\frac{\sigma}{60}
$$

Subsequently, the function $\chi$ becomes

$$
\chi(z, \sigma)=\frac{10}{\sqrt{\sigma+3}}(1-z)^{\frac{\sigma}{60}}
$$

Then, the final new model that describes the evolution of TRIP during bainitic transformation of the 16MND5 steel under low applied stress is

$$
\dot{E}^{t p}= \begin{cases}-\frac{2 \Delta \varepsilon_{\alpha \gamma}}{\sigma_{\gamma}^{y}} \chi(z, \sigma) \ln \left(z_{\ell}\right) \dot{z} \frac{3}{2} S & \text { if } \quad z \leqslant z_{\ell} \\ -\frac{2 \Delta \varepsilon_{\alpha \gamma}}{\sigma_{\gamma}^{y}} \chi(z, \sigma) \ln (z) \dot{z} \frac{3}{2} S & \text { if } \quad z>z_{\ell}\end{cases}
$$


with

$$
\chi(z, \sigma)=\frac{10}{\sqrt{\sigma+3}}(1-z)^{\frac{\sigma}{60}} \quad z_{\ell}=\frac{\sigma_{\gamma}^{y}}{2 \Delta \varepsilon_{\alpha \gamma}} \frac{4 \mu+3 K}{9 K \mu}
$$

The results of the new refined model and experimental results are illustrated by Fig. 9 . It has been found that new predictions achieved by the refined model, Eq. (4.5), are more convenient with experiments than those obtained from the preliminary version of this model for all considerate cases of the applied stress. Indeed, the refined new model is capable to capture not only the fast transformation plasticity observed experimentally at the beginning of the transformation but also the deceleration of this plasticity rate during the second half of the transformation; a profit which cannot be accomplished by the previous models.
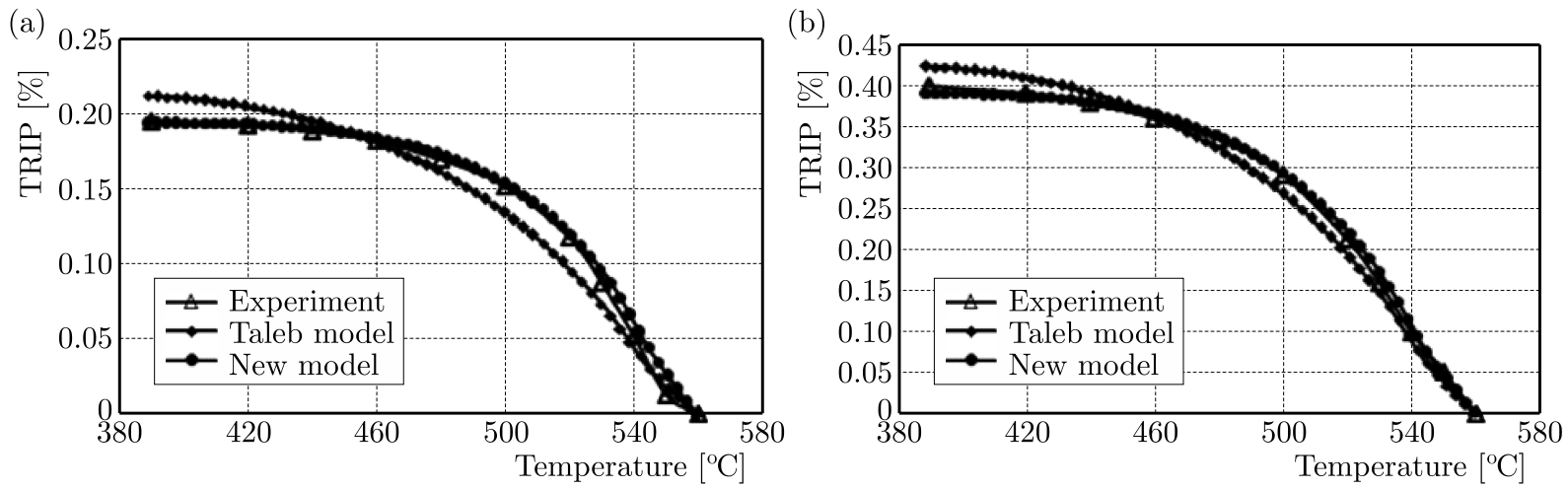

Fig. 9. Transformation plasticity evolution versus temperature during transformation plasticity tests under (a) $30 \mathrm{MPa}$, (b) $60 \mathrm{MPa}$. Comparison between experimental results and predictions of Taleb's and new refined models

\section{Conclusion}

In this study, a new (semi-theoretical) model to predict TRIP induced during bainitic transformation under an external tension loading is developed by upgrading the existing models in the literature. A way to improve these models would be the revising some simplifying assumptions suggested by authors during analysis of the micromechanical approach. Subsequently, new substitute assumptions are reasonably suggested leading then to multiplying Taleb's formula by an appropriate function that is numerically established. The new semi-theoretical model for predicting TRIP produced during bainitic transformation under low tension stress is finally established.

The investigation of the accuracy of the new model is performed in the light of comparison between numerical simulations and experimental results provided in the literature. It has been found that predictions obtained by the refined new model are significantly better than Taleb's forecasts. Furthermore, this new model leads not only to elevate TRIP values at the beginning of the transformation but also to lower them during the second half of the transformation; a result that is experimentally perceived and cannot be described by prior models. Further studies are needed in order to extend this new model for the case of high applied stress and other kinds of transformations.

\section{References}

1. Barbe F., Quey R., Taleb L., Eduardo S.C., 2008, Numerical modelling of the plasticity induced during diffusive transformation. An ensemble averaging approach for the case of random arrays of nuclei, European Journal of Mechanics A, 27, 1121-1139 
2. Coret M., Calloch S., Combescure A., 2002, Experimental study of the phase transformation plasticity of 16MND5 low carbon steel under multiaxial loading, International Journal of Plasticity, 18, 1707-1727

3. DAn W.J., Li S.H., Zhang W.G., Lin Z.Q., 2008, The effect of strain-induced martensitic transformation on mechanical properties of TRIP steel, Materials and Design, 29, 604-612

4. Deng D., Murakawa H., 2013, Influence of transformation induced plasticity on simulated results of welding residual stress in low temperature transformation steel, Computational Materials Science, 78, 55-62

5. Dutta R.K., Amirthalingam M., Hermans M.J.M, Richardson I.M., 2013, Kinetics of bainitic transformation and transformation plasticity in a high strength quenched and tempered structural steel, Material Science Engineering A, 559, 86-95

6. Fischer F.D., Oberaigner E.R., Tanaka K., Nishimura F., 1998, Transformation induced plasticity revised an updated formulation, International Journal of Solids and Structure, 35, 22092227

7. Hoang H., Barbe F., Quey R., Taleb L., 2008, FE determination of the plasticity induced during diffusive transformation in the case of nucleation at random locations and instants, Computational Materials Science, 43, 101-107

8. Leblond J.B., Devaux J., Devaux J.C., 1989, Mathematical modelling of transformation plasticity in steels. I: Case of ideal-plastic phases, International Journal of Plasticity, 5, 551-572

9. Leblond J.B., Mottet G., Devaux J.C., 1986, A theoretical and numerical approach to the plastic behaviour of steels during phase transformations: I - Derivation of general relations, Journal of the Mechanics and Physic of Solids, 34, 395-409

10. Meftah S., Barbe F., Taleb L., Sidoroff F., 2007, Parametric numerical simulations of TRIP and its interaction with classical plasticity in martensitic transformation, European Journal of Mechanics A, 26, 688-700

11. Mohr D., JACQUemin J., 2008, Large deformation of anisotropic austenitic stainless steel sheets at room temperature: multi-axial experiments and phenomenological modeling, Journal of the Mechanics and Physics of Solids, 56, 2935-2956

12. Moumni Z., Roger F., Trinh N., 2011, Theoretical and numerical modeling of the thermomechanical and metallurgical behavior of steel, International Journal of Plasticity, 27, 414-439

13. Shi J., Turteltaub S., Van Der Giessen E., 2010, Analysis of grain size effects on transformation-induced plasticity based on a discrete dislocation transformation model, Journal of the Mechanics and Physics of Solids, 58, 1863-1878

14. Song K.J., Wei Y.H., Dong Z.B., Ma R., Zhan X.H., Zheng W.J., Fang K., 2014, Constitutive model coupled with mechanical effect of volume change and transformation induced plasticity during solid phase transformation for TA15 alloy welding, Applied Mathematical Modeling, 39, 2064-2080

15. Sun C., Fang G., Lei L.P., Zeng P., 2009, Micro-thermomechanical constitutive model of transformation induced plasticity and its application on armour steel, Materials Science and Engineering A, 449, 18-22

16. Tahimi A., Barbe F., Taleb L., Quey R., Gulllet A., 2012, Evaluation of microstructurebased transformation plasticity models from experiments on 100C6 steel, Computational Materials Science, 52, 55-60

17. Taleb L., Cavallo N., Waeckel F., 2001, Experimental analysis of transformation plasticity, International Journal of Plasticity, 17, 1-20

18. Taleb L., Petit S., Julien J.F., 2004, Prediction of residual stresses in the heat affected zone, Journal de Physique IV, 120, 705-712 
19. TAleb L., Sidoroff F., 2003, A micromechanical modeling of the Greenwood-Johnson mechanism in transformation induced plasticity, International Journal of Plasticity, 19, 1821-1842

20. Yankoubi M., Kchaou M., Dammak F., 2013a, Simulation of heat treatment and materials with the use of the Abaqus software, Metal Science and Heat Treatment, 55, 7-8

21. Yaakoubi M., Kchaou M., Dammak F., 2013b, Simulation of the thermomechanical and metallurgical behavior of steels by using ABAQUS software, Computational Materials Science, 68, 297-306

Manuscript received October 9, 2015; accepted for print June 15, 2016 